\title{
$A B C D$ position statement on risk stratification of adult patients with diabetes during COVID-19 pandemic
}

\author{
DINESH K NAGI, ${ }^{1}$ EMMA G WILMOT, ${ }^{2}$ KARISSA OWEN, ${ }^{3}$ DIPESH C PATEL, ${ }^{4}$ LESLEY MILLS, ${ }^{5}$ \\ GERRY RAYMAN, ${ }^{6}$ PETER WINOCOUR, ${ }^{7}$ GOHER AYMAN, ${ }^{8}$ ROHIT PATEL, ${ }^{8}$ CLARE HAMBLING, 9 \\ PRATIK CHOUDHARY ${ }^{10}$
}

\begin{abstract}
At the time of submission of this manuscript, the COVID-19 pandemic had cost nearly 60,000 lives in the UK. This number currently stands at over 120,000 deaths. A high proportion (one third) of these lived with diabetes. The huge acute and emergency medicine effort to support people with COVID19 has had a major knock-on impact on the delivery of routine clinical care, especially for long-term conditions like diabetes.

Challenges to the delivery of diabetes services during this period include a reduction in medical and nursing staff, limitations placed by social distancing on physical clinical space, and balancing virtual vs face-to-face care. There is a need to re-group and re-organise how we deliver routine out-patient adult diabetes services during the ongoing COVID-19 pandemic. We offer some suggestions for how patients can be stratified into red (urgent), amber (priority) and green (routine) follow up with suggestions of how often people should be seen. We also offer recommendation on how we can identify those at highest risk and try and minimise the longterm impact of COVID on diabetes care
\end{abstract}

Honorary Consultant Diabetes and Endocrinology, Pinderfields Hospital, Mid Yorkshire NHS Trust, Wakefield, UK

Consultant Diabetologist, University Hospitals of Derby and Burton,

Associate Professor, University of Nottingham, UK

GP Clinical Lead for Diabetes, Derby and Derbyshire CCG; Partner,

Littlewick Medical Centre, Ilkeston, Derbyshire, UK

Consultant Physician, Royal Free NHS FT, Hon Associate Professor,

University College London, London, UK

Consultant Nurse, Warrington and Halton Teaching Hospitals NHS FT, UK

6 Diabetes Department, The Ipswich Hospital and Ipswich Diabetes Centre and Research Unit, East Suffolk and North Essex NHS Foundation Trust, Colchester, UK.

QEIl Hospital, Welwyn Garden City, UK

Diabetes UK

9 Department of Public Health and Primary Care, School of Clinical Medicine, Cambridge, UK

10 Professor of Diabetes, Diabetes Research Centre, University of Leicester, Leicester General Hospital, Leicester, UK; Chair, Diabetes Technology Network, UK

Address for correspondence: Dr Dinesh Nagi

Honorary Consultant Diabetes and Endocrinology, Pinderfields Hospital, Mid Yorkshire NHS Trust, Aberford Road, Wakefield, West Yorkshire

WF1 4DG, UK

E-mail: D.nagi@nhs.net

https://doi.org/10.15277/bjd.2021.282
During the COVID pandemic we have seen things happen in days that previously took years. The restart of diabetes services has triggered a more widespread use of virtual consultations and data management systems, but also offers an opportunity for more joined-up and cohesive working between primary and specialist care. While we do our best to keep our patients and colleagues safe, this pandemic is already proving to be a catalyst for change, accelerating the appropriate use of technology in diabetes care and implementing innovative solutions. To achieve this aspiration, further work - currently led by the Association of British Clinical Diabetologists in collaboration with Diabetes UK and the Primary Care Diabetes Society - to make recommendations on future proofing diabetes care in UK is in progress. Br J Diabetes 2021;21:123-131

Key words: COVID-19 pandemic, risk stratification, diabetes services, virtual consultations, recommendations.

\section{Introduction}

As the COVID-19 pandemic made its way to the UK, clinical services were called to action with a clear priority of taking care of the many who suffered from this condition. Within days, almost all routine clinical care in the UK was suspended, with many diabetes healthcare professionals called up to front-line duties. Work around diabetes and COVID-19 initially centred on keeping people safe, with development of practical recommendations to manage people with diabetes and COVID-19. ${ }^{1-3}$ Government advice for people with diabetes was to stay at home as much as possible and follow strict social distancing ${ }_{1}^{4}$ but they were not initially included in the highest risk shielding group. Despite this advice, we know that almost a third of the people who died during this pandemic in the UK had diabetes, and diabetes was associated with a 2-3-fold excess risk of dying during this period. ${ }^{5}$

In the UK, indications were that we were through the worst of this initial first phase, ${ }^{6}$ but the pandemic has continued and we are now amidst a prolonged second and third wave of this pandemic, with hospital services once again at breaking point.

During this we see no choice but to develop an urgent recovery plan that not only prioritises those who are most vulnerable, but also minimises the risks of 'indirect' harm due to postponement or cancellation of non-urgent and routine medical care, and conforms to new guidelines around social distancing and our ability to use our physical clinics. 
The 'restart' of services provides an opportunity to redevelop existing services for the better. These recommendations from the Association of British Clinical Diabetologists (ABCD) are designed to stimulate discussions and provide a guidance for the short and medium term recovery, and provide a glimpse into how this COVID-19 pandemic may end up changing diabetes services for ever. An interim and concise summary of the $A B C D$ recommendation for risk stratification of adults with diabetes has recently been published. ${ }^{7}$

\section{Key challenges during recovery}

1. Recovering missed activity: Across the country, thousands of appointments across primary and secondary care have been cancelled or postponed. Referrals to specialists, access to eye screening, annual reviews, insulin pump upgrades, starts on new medications such as glucagon-like peptide-1 (GLP-1) analogues or insulin or new technologies such as insulin pumps and FreeStyle Libre sensors have been reduced or delayed. Recovering this lost ground will put a great deal of strain on systems. The most vulnerable patients will be at greatest risk of getting lost in the system.

2. Ongoing reduction in staffing: It is possible that, in the coming months, many specialist staff including consultants, specialist nurses and trainees who delivered routine outpatient diabetes care will remain under increased pressure to cover general internal medicine and inpatient diabetes. Trusts may require staff to take most if not all of annual and study leave allowances during 'non-GIM' blocks of work, further reducing face-to-face outpatient capacity. Primary and community care services are also likely to face workforce challenges.

3. Social distancing in clinic: New social distancing norms will result in significant reductions in the number of patients we can see in outpatients and other clinical settings, and we will need to adapt working processes to minimise risks to patients, ourselves, our healthcare and administrative staff. Waiting areas may need to be re-designed to manage bottlenecks such as reception, phlebotomy, nurse assessment areas as well as clinic areas used for diabetes education, technology group starts and technology uploads. This could result in a $25-50 \%$ reduction in availability of clinical space with huge variability from trust to trust. In primary care, adaptations due to social distancing may be even more challenging.

4. Delayed presentations: The increased needs of people whose complications or care of their diabetes may have worsened during the pandemic. Anecdotally, we are now seeing more people coming into hospital with worsened complications (eg, foot disease) or seeing more people being discharged with insulin or new management plans that may not have appropriate community support.

5. Restarting group interventions: Social distancing will mean changes to how group interventions such as structured education or starting new treatments such as insulin, GLP-1 analogues or insulin pumps and continuous glucose monitors are delivered. While some people will welcome an opportunity for virtual support rather than having to travel to hospital, some may find this more difficult. We must consider accessibility for those who are not IT enabled, otherwise we risk increasing healthcare inequality further.

6. Education and training: The move of activity from face-toface to virtual will affect how we deliver training, education and supervision for colleagues.

The impact of all these factors is a net increase in demand superimposed on a reduced capacity to provide care, with delays in delivery of care and ultimately a reduction in the quality of care, adding more complexity to the need for diabetes care. All this at a time when the data have shown that those living with diabetes are at the highest risk of mortality from COVID-19 and glycaemic control seems to be a predictor of outcomes. ${ }^{8}$

\section{Roadmap to recovery}

Five general principles laid out by the Academy of Medical Royal Colleges related to patients and staff provide an operating framework for the recovery plan. ${ }^{9}$

- Principle 1 - People with diabetes should be offered virtual or remote care where safe and possible.

- Principle 2 - Patients should be offered alternative management options which are temporary or permanent using shared decision making.

- Principle 3 - Patients should feel safe and protected when they need to access direct healthcare in all settings.

- Principle 4-Staff should feel enabled, safe and protected to deliver care.

- Principle 5 - Staff should be supported and provided with training and education that will ensure adequate preparation of current and future staff.

The recovery plan should start and focus on:

1. Risk stratification and triage

2. Resource allocation

3. Transfer of care to specialist centres when required

4. Communication prioritisation of those most in need of clinical support

5. Maintaining social distancing

6. Ensuring ongoing access to evidence-based interventions

7. Ensuring seamless communication and collaboration between primary and secondary care services

\section{Risk stratification and triage}

As mentioned previously, up to one-third of appointments over the past 3 months may have been cancelled or postponed, and the number of appointments available in the next 6 months may be significantly lower than usual. As services move into a recovery phase, it will be important to prioritise people most at risk, especially in light of risk factors as demonstrated in recent national NHS England data. ${ }^{5}$

We propose multi-factorial risk profiling. Services should aim to see those in the urgent category within the next 3 months and those in the priority group within 6 months. Those in the routine group should be sent correspondence providing them with educational resources and access to helpline numbers so they can make contact with the team if their circumstances change. 
We acknowledge that the ability to deliver this will depend upon available capacity of teams both in specialist and primary care. A limited capacity, for example, which is reduced to $25 \%$ may mean only acute and emergency care can be provided.

URGENT "Red": These people with diabetes should be reviewed as soon as possible (within 3 months) by their team and require a detailed individualised care plan to address their risk factors and minimise their risk of admission. We estimate this may make up $10-20 \%$ of those in specialist services. These should include those with newly diagnosed or possible type 1 diabetes.

- $\mathrm{HbA}_{1 \mathrm{c}}>86 \mathrm{mmol} / \mathrm{mol}(10.0 \%)$

- Uncontrolled BP (>160/100)

- Known chronic kidney disease level $\geq 4$ (estimated glomerular filtration rate (eGFR) $<30 \mathrm{~mL} / \mathrm{min}$ )

- High risk of hypoglycaemia: severe hypoglycaemia in the last year or complete loss of hypoglycaemia awareness

- High risk of admission: recent admission with diabetic ketoacidosis/hyperglycaemic hyperosmolar syndrome or a diabetesrelated co-morbidity such as ischaemic heart disease, heart failure or cerebrovascular disease

- Active diabetes foot disease

- Planning pregnancy in the next 6 months

PRIORITY "Amber": These people should be reviewed within the next 6 months by their team:

- People with $\mathrm{HbA}_{1 \mathrm{c}} 64-86 \mathrm{mmol} / \mathrm{mol}(8.0-10.0 \%)$

- Elevated hypoglycaemia risk such as those with impaired hypoglycaemia awareness or those with $\mathrm{HbA}_{1 \mathrm{c}}<48 \mathrm{mmol} / \mathrm{mol}$ $(<6.0 \%)$ on insulin or sulfonylureas, with known frailty, cognitive impairment

- Those with known chronic kidney disease stage 3B (eGFR $<45$ $\mathrm{mL} / \mathrm{min}$ or current albuminuria) or rapidly declining eGFR (>15 $\mathrm{mL} /$ year) or progressive albuminuria (albumin to creatinine ratio $>30$ )

- Known 'high-risk' feet not known to podiatry service

- Those with no diabetes review in the last 15 months

Where capacity allows, these further groups can also be considered in the priority "amber" group:

- Body mass index (BMI) $>40 \mathrm{~kg} / \mathrm{m}^{2}$ (or BMl $>35 \mathrm{~kg} / \mathrm{m}^{2}$ associated with co-morbidities)

- Those aged $<40$ years with known early onset complications (type 1 or type 2 diabetes)

- Those not meeting their three treatment targets $\left(\mathrm{HbA}_{1 c}, \mathrm{BP}\right.$, lipids) at the last visit

ROUTINE "Green": Given the challenges encountered during the first and subsequent waves of the pandemic, it is possible that the patients in this category may not be seen well into 2021, posing significant concerns. We suggest that health care providers should contact these people explaining the situation and providing them with information and resources, with guidance on what to do if any of their parameters change. For example, if they become unwell or their blood glucose readings are much higher or much lower than usual. They should be advised to monitor their glucose where appropriate (insulin treated patients) and monitor their weight and blood pressure where appropriate. Signpost individuals to educational resources commissioned by CCGs and the new NHSE educational websites: ${ }^{10}$

- $\mathrm{HbA}_{1 \mathrm{c}}<64 \mathrm{mmol} / \mathrm{mol}$ (8.0\%) with no risk factors for hypoglycaemia

- Stable renal function (eGFR >60 mL/min; no current microalbuminuria or proteinuria)

- People with type 2 diabetes meeting all three treatment targets in the last 12 months (BP $<140 / 80$, total cholesterol $<5 \mathrm{mmol}$, $\left.\mathrm{HbA}_{1 \mathrm{c}}<58 \mathrm{mmol} / \mathrm{mol}(<7.5 \%)\right)$ with low-risk feet

Special considerations: There are of course some people with diabetes who will fall outside these recommendations who may be considered high risk (people with learning difficulties, social difficulties, mental health issues, frailty, autonomic neuropathy), or those with cystic fibrosis-related diabetes, post-transplant diabetes or who have other co-morbidities that put them at high risk. It is important to recognise that often those in the high-risk group with associated mental health, learning, social or personality conditions may be the least likely to engage with services at this time. ${ }^{11}$ There may be some patients in the high-risk group who the team know well and who are 'stable' in the high-risk group and where previous attempts to engage and support have been unsuccessful.

Other special needs groups are individuals discharged from hospital after a COVID or non-COVID-related admission who may require monitoring and support due to unstable glucose levels or because they have been started on insulin, and those who need assessment and optimisation of their diabetes prior to elective surgery. Teams will need to make individual case-based decisions on where to prioritise, including the appropriate time frame for follow-up, depending on their own resources in these circumstances. The National COVID-19 group has produced guidance for a supportive discharge which is now available. ${ }^{12}$

Use of IT to help risk-stratify patients (Figure 1): A key barrier to supporting those most in need is identifying them in the first place. Diabetes services with a diabetes database will be able to run searches to identify those most in need according to the criteria for risk stratification. Primary care IT systems can run data-driven searches $\left(\mathrm{HbA}_{1 \mathrm{C}}, \mathrm{BP}\right.$, lipids, date of last annual review or foot check), but some specialist services may not have this option. If such systems are not in place, we suggest that alternatives should include the following:

- Using Primary Care Network, CCG or STP (using local GP databases) capabilities to coordinate the search and reporting of outcomes on patients in the red category and to allow the tracking of patients in the red, amber and green categories.

- Identification of those in need through glucose data in the cloud (eg, LibreView, Clarity) which can rank all patients in the service by estimated time in range, estimated $\mathrm{HbA}_{1 \mathrm{c}}$, time below range, etc.

- Asking the laboratory to generate a priority list using $\mathrm{HbA}_{1 c}$ results.

- Consider options available if local review of cases is the only 
Figure 1. Flow chart of risk stratification

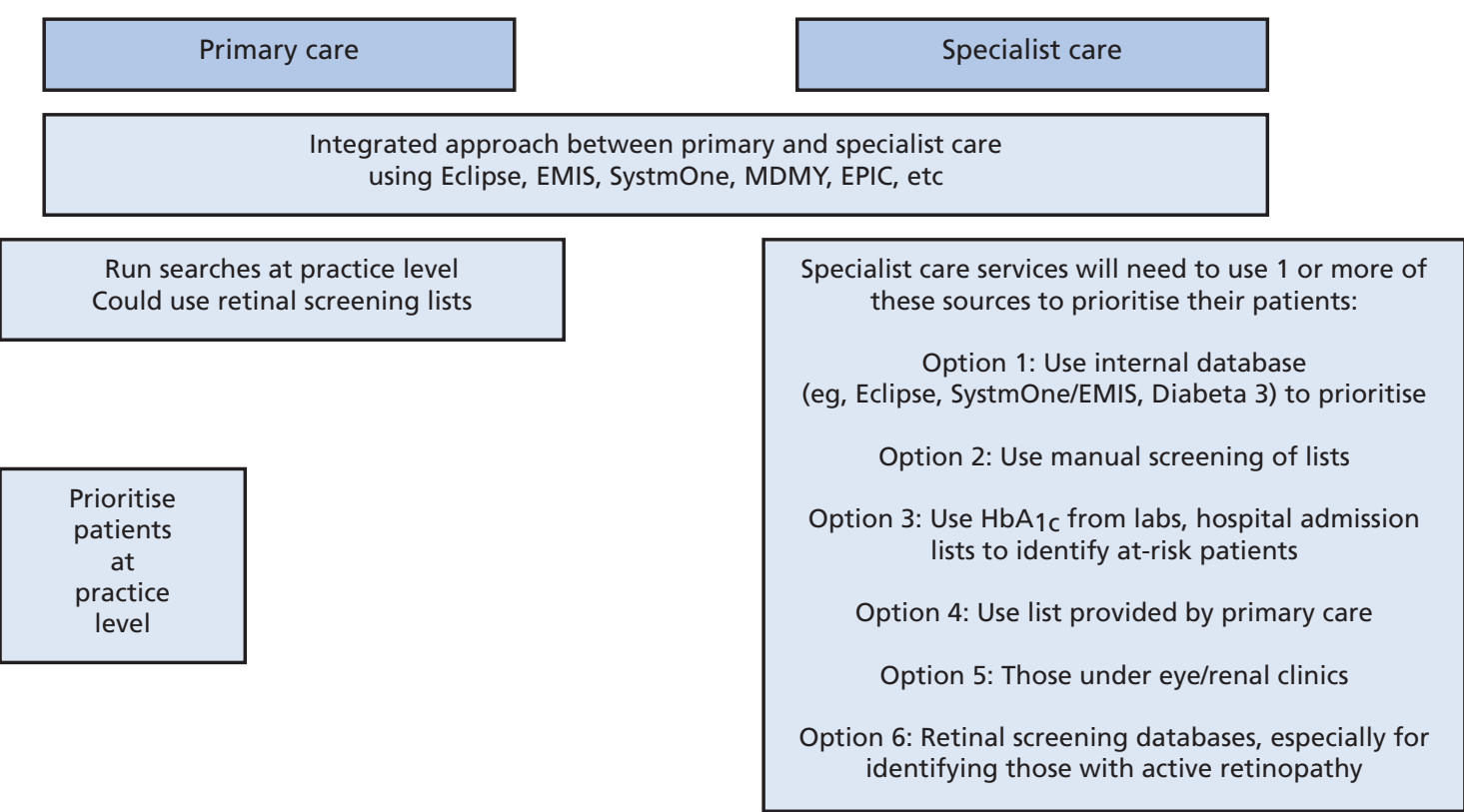

option; administrative staff, medical students and trainees may be able to offer support.

- Diabetes teams are generally aware of the people with diabetes who require frequent support to manage their diabetes and this information should be used to recall and prioritise patients (recurrent diabetic ketoacidosis admissions, recurrent severe hypoglycaemia, etc).

\section{Resource allocation}

We need to re-shape how we allocate both our human and physical resources during the recovery phase. As people are triaged, there are number of factors that should be kept in mind to maximise the utilisation of our resources. Things that need to be considered are making sure the person with diabetes sees the right clinician in the right environment at the right time.

- Right clinician: We need to make sure the person with diabetes sees the most appropriate clinician for their individual needs (doctor, specialist nurse, dietitian, psychologist) who needs to provide holistic care and gather ALL relevant information and metrics - need to minimise the number of face-to-face contacts for infection control and avoidance of duplication and/or unnecessary recall.

- Right environment: We also need to make sure that we assess if the individual needs a virtual or face-to-face appointment this may depend on clinical need (eg, need for foot examination or education) or on individual preference.

- Right time: Appointments should become more needs-based. In the initial recovery phase we would recommend the suggested triage system, but moving forward the team should consider how many appointments the person is likely to need in the next 6 months based on their needs. This will help with planning on capacity utilisation. A suggested frequency is shown in Table 1, but this may have to be modified depending on locally available clinic capacity.

Multidisciplinary team (MDT) review of cases and appropriate caseload allocation may be a wise investment of time that increases efficiency in clinic. This may allow appropriate allocation of individuals to clinics and clinicians and allow 'virtual' decision making, review and handover between members of the diabetes team. Thinking through patient flows to optimise data collection (bloods, measurements such as height and weight, questionnaires and download of devices) before the patient is seen can help make clinics more efficient. Pathway coordinators, as used in other specialties, may allow more optimal use of resources and ensure that every contact counts. In particular, there is a recognition that in some

Table 1 Suggested annual follow-up requirements

\begin{tabular}{|c|c|c|c|}
\hline & RED & AMBER & GREEN \\
\hline Face-to-face & $\begin{array}{l}\text { May need at } \\
\text { least } 1\end{array}$ & $\begin{array}{l}\text { May not be } \\
\text { needed }\end{array}$ & $\begin{array}{l}\text { May not be } \\
\text { needed }\end{array}$ \\
\hline $\begin{array}{l}\text { Virtual or } \\
\text { face-to-face } \\
\text { as per need }\end{array}$ & $4-6$ & $2-4$ & 1 \\
\hline $\begin{array}{l}\text { Telephone/email/ } \\
\text { text messages }\end{array}$ & $\begin{array}{l}\text { At least } 1 \\
\text { face-to-face } \\
\text { appointment }\end{array}$ & $\begin{array}{l}\text { Need-based } \\
\text { between } \\
\text { virtual } \\
\text { appointments }\end{array}$ & $\begin{array}{l}\text { For rapid } \\
\text { access } \\
\text { support } \\
\text { as needed }\end{array}$ \\
\hline \multicolumn{4}{|c|}{$\begin{array}{l}\text { The mode of follow-up is dependent on patient choice, but we would expect that } \\
\text { over a population which excludes antenatal and diabetic foot services, the } \\
\text { remaining } 70 \%+\text { of "Green" appointments could all potentially be virtual. These } \\
\text { contacts should be needs-driven. }\end{array}$} \\
\hline
\end{tabular}


places, while doctors have been rostered to different acute medical rotas, the specialist nurses may have worked in isolation and this needs to be addressed urgently to recover MDT working for safeguarding good quality care.

Clearly, during these times where overall team capacity can be severely compromised, we recommend that the guidance previously produced during the first wave by the $A B C D$ jointly with the Royal College of Physicians of London (RCPL) and the Society for Endocrinology (SfE) is followed (see Table 2). ${ }^{13}$

Flexibility of virtual clinics - changing the way we work: It is possible to conduct virtual consultations from home, reducing the need for clinicians to travel into work, facilitating social distancing within the diabetes department and allowing clinicians who may be shielding due to their own risk factors to contribute. This opens up options for more flexible working enabling colleagues to work flexibly, allowing for school runs or virtual evening clinics for those who prefer them. NHS England has published some guidance on virtual clinics. ${ }^{14}$ User involvement in the redevelopment of services is critical and online surveys may facilitate this.

Optimising working with other specialities: It is important that we have an integrated approach across primary and secondary care as well as other specialties to minimise duplication and support joint decision making. Removing the need to be in the same physical space, it is now much easier to be in the same virtual space and joint appointments with patients with colleagues from cardiology, renal or mental health teams or even with primary and specialist providers will be valuable to improving the quality of care. This will require collaboration and a systems approach.

Moving away from routine to 'as required' diabetes reviews: Historically, diabetes services have provided ongoing regular support for individuals focusing on care processes, with little regard for the level of need of an individual or of clinical priority. Current pressures require services to evolve from this model into a system which allows rapid access for those most in need. How this is delivered will vary by location, but this is a much needed development.

Rethinking the annual review care process: Although completion of the key care processes remains the cornerstone of good diabetes management, completion of these has been variable, ${ }^{15}$ with only $41 \%$ and $58 \%$ of those with type 1 and type 2 diabetes, respectively, completed before the COVID-19 pandemic. ${ }^{16}$ It is anticipated that completion of annual review process is likely to be much lower in 2021/2022, due to the clinical pressures on team and the suspension of QOF this year. As a result of QOF, care processes have historically been viewed as the responsibility of primary care.

We now enter a new era where every contact must count. Every professional working in diabetes has a duty of care to make the most of our limited resources and ensure that key care processes are completed as far as is practical in the individual's consultation. Collecting and sharing these key data, irrespective of where the individual is seen (primary or specialist care), will avoid duplication and reduce the need for multiple appointments with face-to-face contact.

Rapid access to services: Over the next few months, as we try to get our services back on track, there is the possibility that those whose reviews have been delayed may require urgent support. It is

Table 2 Delivering diabetes and endocrine services in acute NHS Trusts during COVID-19 pandemic reset

\begin{tabular}{ll}
\hline Disease condition \\
$\begin{array}{ll}\text { At } \mathbf{2 5} \% \text { of capacity (a) } \\
\text { Inpatient } & \text { (1) Emergency inpatient: acute metabolic emergencies } \\
& \text { (2) Urgent inpatient diabetes care - on other wards } \\
\text { Outpatient } & \text { (3) Urgent and acute diabetes foot disease (inpatient and outpatient) } \\
& \text { (4) Antenatal clinic diabetes care }\end{array}$
\end{tabular}

At $50 \%$ of capacity (b)

(a) and in addition

Outpatient (5) New referrals: urgent new type 1 diabetes, insulin initiation, urgent technology support, some face-to-face

\section{Comments}

Smaller units may struggle to provide these services at $25 \%$ of capacity

At $75 \%$ of capacity (c)
(a)+(b) and in addition
(6) Inpatient support for less urgent diabetes patients on other wards renal, retinal)
(a) $+($ b) $+(c)$ and in addition
(1) Full clinical services depending on local arrangements
(2) Diabetes follow-up review by current case mix
(3) Primary care support including full integrated care
(4) Joint clinics
(5) Teaching and training (including patient education)

(7) Complex type 2 diabetes (poor metabolic control and/or complex comorbid indications - Many will need face-to-face

At $\mathbf{9 0}-\mathbf{1 0 0} \%$ of capacity

Triage and consult by a clinician according to risk stratification and patient need

Dr Peter Winocour, Professor Stephanie Baldeweg, Dr Dinesh Nagi: on behalf of the Association of British Clinical Diabetologists (ABCD) and the Society for Endocrinology (SfE) and Joint Specialist Committee (JSC) for Diabetes and Endocrinology at RCP London

Diabetes mellitus (included after permission from authors). 
critical that there is a pathway for them to access urgent support and advice. Examples may be rapid de-stabilisation due to intercurrent illnesses or new treatments, or those who need particular support around elective surgery. We need to ensure that the provision of timely care for this group is taken care of, despite even a limited capacity of individual teams.

\section{Transfer of care to specialist centres when required}

As clinical reviews restart, particularly in primary care, it is important that we review and optimise the process of referrals to specialist centres. With virtual reviews, virtual MDTs and advice and guidance systems, it would be ideal for specialist services to offer timely and valuable advice to primary care colleagues. Doing this using shared IT systems may reduce the number of referrals, but also speed up the delivery of advice and how soon appropriate referrals are seen in specialist care. This should become a norm for the future.

\section{Communication prioritisation of those most in need of clinical support}

As we develop the recovery strategy for diabetes services, a clear local plan, which includes early communication to people with diabetes will be essential. People with diabetes, especially those at high risk (high $\mathrm{HbA}_{1 c}$, those with diabetes related complications, special needs and special groups) require early contact to inform them about these local plans for supporting these individuals, to ensure that they stay safe and healthy. Informing them that there are provisions for rapid access to seek urgent support from GPs in the primary care and also from the Diabetes Specialist team will be vital during this crisis and provide much needed re-assurance.

\section{Maintaining social distancing}

This is a key challenge for both primary and specialist care, with a huge impact on the numbers of patients that can be seen face-toface. Strategies to maintain social distancing in clinics may include alternating face-to-face appointments with virtual ones, or reducing the number of clinicians doing face-to-face clinics at any given time. In these instances, it is important to emphasise to individuals attending that social distancing will be maintained.

Many teams have done this through implementation of virtual or video clinics $^{17}$ using telephones or NHS-approved video systems such as NHS Attend Anywhere, AccuRx or MyClinic. The virtual consultations have worked well; however, healthcare professionals as well as people with diabetes will take some time to get used to these new ways of interacting..$^{18,19}$ It is perhaps important to reassure patients that quality of consultation is not reduced and, where required, face-to-face appointments are made available.

We recognise that, while this is an opportunity to work together and in innovative ways, we must not inadvertently increase the health inequalities which already exist in the NHS due to the lack of access to digital platforms in a significant proportion of people with diabetes. An assessment of the most appropriate form of contact will need to be undertaken on an individual level.

Optimising virtual consultations: There is invariably a leadin phase as we all learn to use virtual technologies. Virtual consul- tations will not cover every possible scenario in clinical practice and it is not for everyone, but when it works it can replicate the physical consultation processes in the virtual space. However, we need to be aware that some in the most high-risk groups are individuals who may be most likely to have very limited or no access to online technology, or possibly even a safe environment in which to conduct a remote clinical interaction. Some key principles for video consultations are outlined in Box 1. Excellent guidance is also available from the Primary Care Diabetes Society. ${ }^{19,20}$

Prior to virtual clinics, having appropriate access to data is key and the following tips may be valuable:

Access to glucose data: Data management platforms that collect glucose and insulin data such as Diasend, Carelink, LibreView and Clarity are key for virtual consultations. Ensuring people are connected and have access to information to allow them to upload data becomes vital. In the absence of this, we can still rely on skills we use in our face-to-face appointments such as asking the patient to read through their meter as we jot down the results to try and see patterns in the data, or e-mailing the results to healthcare professionals.

\section{Box 1 Guidance for remote consultations}

- Administration: make sure the person with diabetes knows this is a virtual appointment and to be ready to accept a message inviting them to a virtual consultation or a call from a 'withheld' number as appropriate. This requires some planning and coordination.

- Privacy and consent: Ensure the person you are consulting with is comfortable with this means of contact (telephone is always there as back-up) and you are both in a quiet and private area where confidentiality is maintained. System approved by the local NHS organisation should be used (e.g. Attend Anywhere, Accurx).

- Technology: Ensure the Wi-Fi, picture and sound are all working and of adequate quality.

- Consulting: Use a computer screen with high resolution rather than a phone to help pick up on the usual non-verbal ques; try to ensure the camera is at eye level but sit back - leaning forward can come across as being too intense, intrusive and even intimidating. Platforms such as Attend Anywhere allow patients to nominate another person to join their appointment even if living in another household and therefore cannot join the call from the same room where the patient is. The nominated person can be invited to join the consultation through text or email and it is important to replicate the situation where you might usually accompany, for example, a sight-limited or elderly relative to their appointment and help improve their understanding of that consultation. This can also be important for translation support for those whose main language is not English.

- Location: Virtual clinics could potentially be done from non-clinical areas like offices or even from home to minimise risk to healthcare professionals and reduce footfall in clinical areas, and even allow clinicians to work from home to minimise occupancy in what are often crowded clinical or office spaces. Alternatively, virtual reviews could be interspersed with essential face-to-face consultations to minimise footfall in clinics.

- Emotional connection: Try to avoid the consultation becoming too didactic and data centred. Start with open questions and reflections on positive achievements. Often, emotional connection can be challenging. 
Access to other clinical data:

- Home blood pressure checks (temporary loan of BP machines or self-purchase)

- Smoking status

- Home/linked weighing machines to facilitate weight management

- Providing persons with diabetes with a way to provide data that is self-reported (hypoglycaemia awareness scores or diabetes distress scores).

- Use of health data monitoring apps such as Apple Health Kit or systems such as FLORENCE telemedicine

- Use of mobile/community phlebotomy to minimise the physical need for patients to attend hospital

- Showing people how to foot self-check ${ }^{21}$

\section{Ensuring ongoing access to evidence-based interventions}

Structured education: Programmes such as Dose Adjustment for Normal Eating (DAFNE) have been shown to be cost effective ${ }^{22}$ and to improve glucose control while reducing hypoglycaemia. ${ }^{23}$ Importantly, they also significantly reduce diabetes distress, and the group component of this has been key. Some online education portals are available, such as BERTIE online and My Diabetes My Way10 and the newly commissioned NHS England MyType1Diabetes. We can explore whether an intervention such as DAFNE, which relies on interaction between participants and between the educator and the participant, will work in the virtual space.

Type 1 diabetes technology (insulin pump, continuous and flash glucose monitoring): Several centres have already started

Figure 2. Technology: insulin pump, continuous and flash glucose monitoring pathway

\section{Flow chart for commencing CGM remotely}

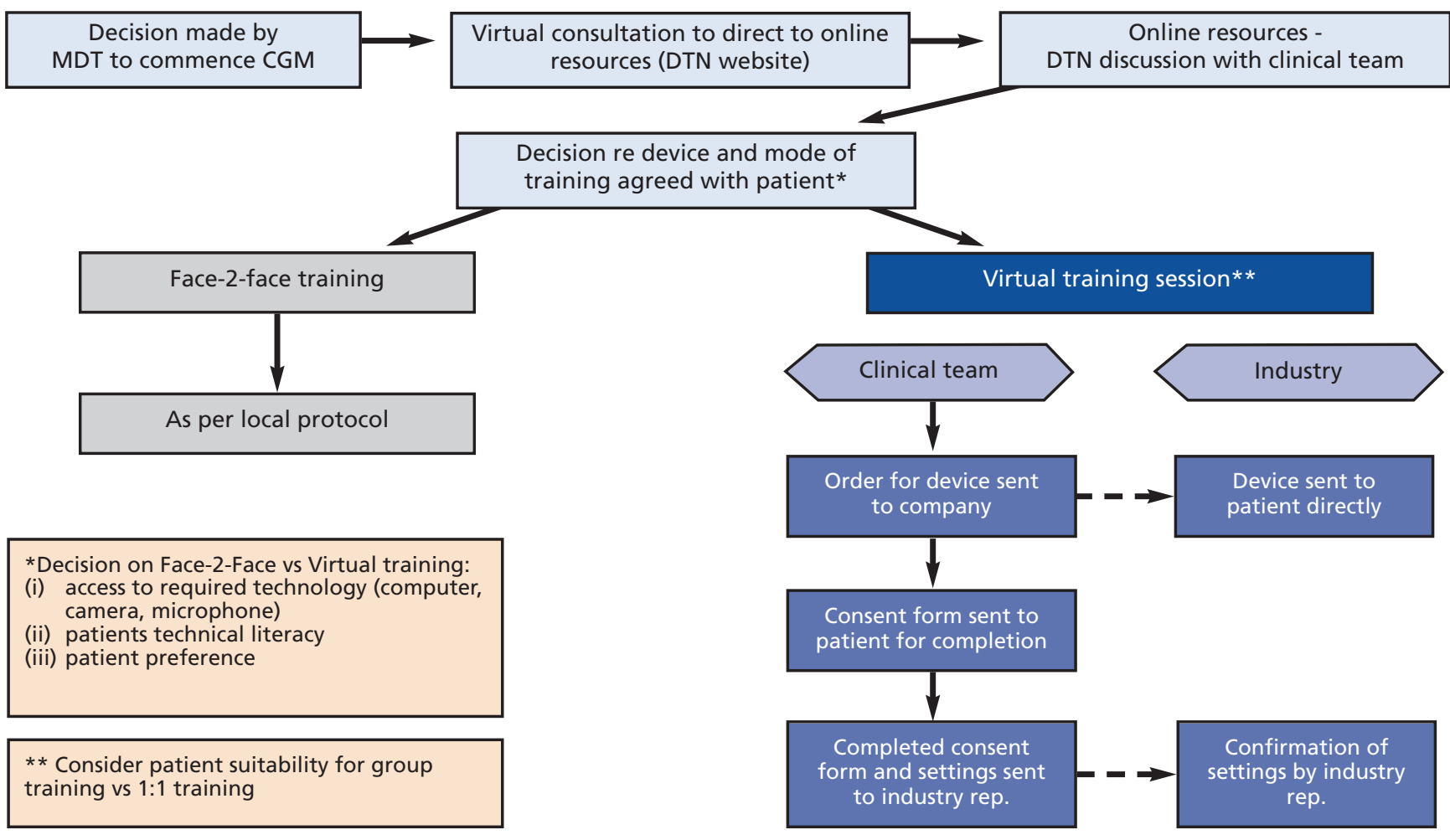

Date/time of virtual training session agreed with patient

Clinical training with DSN

Checklist completed
Technical start with industry rep.

Checklist completed 
initiation of Freestyle Libre or continuous glucose monitoring virtually. We propose a pathway that allows virtual starts for all technology (Figure 2).

Insulin pump upgrades and renewals: It is important that patient choice is not sacrificed. The person living with diabetes needs to interact with their pump 5-10 times a day, and it is paramount that the choice of device best meets their needs. Diabetes Technology Network UK plans to develop a virtual showroom of all options to allow this.

Type 2 diabetes injectable therapies: The timely escalation of therapies has never been so paramount. Remote starts of both insulin and GLP-1 analogue therapy are possible and are happening in many places.

\section{Ensuring seamless communication and collaboration between primary and secondary care services}

Communication within teams: Clear communication of the recovery strategy, ensuring all team members are working to the same goals, is important. This is critical to resource utilisation, making sure the right patient sees the right clinician.

Communication between teams: Rapid and effective communication between primary and secondary care is key. Investment and thought into communication strategies to optimise the process for advice and guidance, as well as referrals and discharges between primary and specialist care, will help maximise our care and target resources to those who need them most. Coordinating appointments between specialists when multiple teams are involved is important.

Integrated IT systems can facilitate this real-time communication on the individual patient level (eg, ECLIPSE, SystmOne, EMIS, MyDiabetesMyWay, EPIC). Adoption of these systems is critical in moving forward into a new era where data-driven prioritisation of care can help us offer the right treatment to the right patient at the right time. As we move into the recovery phase, it is crucial that primary and secondary care teams work together to increase efficiencies and reduce duplication, and integrated IT systems allow a number of benefits that support all the above points including risk stratification, appropriate transfer of care, resource utilisation and optimising virtual clinics. Ensuring that individuals with diabetes are part of these discussions whenever possible and are kept informed at all times is crucial.

- Clinicians can see the full clinical record, facilitating specialist remote management and reducing the need for face-to-face review.

- Allows for the rapid escalation and de-escalation of care, supporting prioritisation of those most in need in a timely manner.

- Supports virtual working by providing clinicians with access to information such as repeat prescription templates, retinal screening outcomes, discharge letters from other local clinical teams including neighbouring hospitals.

- Creates a system-wide database to facilitate case prioritisation and audit of outcomes.

- Immediate communication between primary and secondary care clinicians for advice and change of medication.

\section{The opportunity: the right treatment to the right person at the right time in the right way}

COVID-19 has already had a significant adverse impact on those with diabetes. We need to work to ensure further harm is avoided and further lives are not lost. As we reshape our services including a shift to virtual systems to protect people with diabetes, we have an opportunity over the next 6-12 months which will have a lasting legacy on the way we provide care to people with diabetes.

The use of technology to provide that care in the least disruptive way will be a valuable legacy of the COVID pandemic. We must use this restart to revamp the way we deliver care. We must learn how to use the routine data to identify those at the greatest risk and help support them in the way that suits them best.

The move to databases, integrated care models and virtual clinics may herald in a new more responsive way in which we support people living with diabetes, in a way we have not done before. Finally, we suggest that this is a golden opportunity to learn from the crisis and have highlighted some of the benefits of the new ways of working.

The recent publication of the National Getting It Right First Time (GIRFT) diabetes report ${ }^{24}$ has highlighted the importance of maintaining vital inpatient diabetes teams to ensure the best possible care to those admitted to hospital with COVID-19, using guidelines produced by the National COVID-19 Group with support from the $A B C D .{ }^{12}$

\section{Recommendations}

- Planning the recovery of lost diabetes activity should remain an urgent priority for diabetes teams in both primary and specialist settings.

- We recommend an integrated approach where primary and specialist teams support each other across sectors, with a joined-up approach to maximise available opportunities to ensure an 'every contact counts' ethos, avoiding duplication of activity.

- Teams should use available datasets to risk stratify their patients to ensure they prioritise review of those with the greatest need. This is likely to require greater IT and administrative support.

- IT systems need to be available, integrated and accessible across the whole healthcare system to deliver seamless care.

- We strongly support the use of virtual consultations in delivering diabetes care, recognising that we cannot completely replace face-to face consultations which will be important in certain clinical situations.

- We recommend a 'Right person, Right place, Right time' approach to follow-up that takes into account individual care requirements, co-morbidities and personal preferences to determine the timing, frequency and location of follow-up rather than the routine follow-up model of care.

- Communication within and across teams remains vital at times like this, but timely and clear communication with the person with diabetes will ensure that we avoid confusion, duplication and chaos while recovering lost diabetes activity during COVID19.

- It is crucial that the medical workforce is adequately supported as many colleagues will be mentally and physically 


\section{Key messages}

- The ongoing COVID-19 pandemic has posed a serious disruption to the diabetes services in the UK

- $A B C D$ guidance is designed to help and support diabetes teams to develop a local strategy to plan the recovery of lost clinical activity

- It is important that those at the highest risk are supported to ensure that they stay safe during the pandemic

- Lessons learnt during the pandemic will help shape diabetes services in the future.

- We must ensure that key innovations (i.e virtual consultation) learnt during this crisis, are not lost and must be at the front of our mind while reshaping clinical services

exhausted from provision of frontline services during the COVID crisis.

- $A B C D$ is fully supportive of the National GIRFT recommendations, especially for maintaining in-patient diabetes teams during this crisis. ${ }^{24}$

Author contributions DKN, PC, PW and EGW conceived the paper. $\mathrm{KO}, \mathrm{DP}, \mathrm{LM}, \mathrm{GR}$ and $\mathrm{CH}$ provided input and reviewed the manuscript. GA and RP were patient representatives from Diabetes UK who provided valuable input to the discussion and reviewed the manuscript. PC and DKN wrote the drafts.

Conflict of interest PC has received personal fees from Novo Nordisk, Lilly, Sanofi, Abbott UK, Dexcom, Medtronic, Insulet and Novartis. EGW has received personal fees from Abbott Diabetes Care, Dexcom, Eli Lilly, Insulet, Medtronic, Novo Nordisk and Sanofi Aventis.

\section{Funding None.}

\section{References}

1. Bornstein SR, Rubino F, Khunti K, et al. Practical recommendations for the management of diabetes in patients with COVID-19. Lancet Diabetes Endocrino/ 2020;8(6):546-50. https://doi.org/10.1016/S2213-8587(20)30152-2

2. NHS England, Association of British Clinical Diabetologists, Royal College of Physicians. Clinical guide for the management of people with diabetes during the coronavirus pandemic. 2020 (accessed 11 April 2020). https://abcd.care/announcement/delivering-diabetes-and-endocrine-services-acute-nhs-trusts-during-covid-19-pandemic

3. Nagi DK, Choudhary P, Wilmot E, Winocour P. Supporting people with diabetes during the COVID-19 pandemic without face-to-face appointments. Br J Diabetes 2020;20(1):1-4. https://doi.org/10.15277/bjd.2020.246

4. GOV.UK. 2020. Guidance National Lockdown: Stay at home. https://www.gov.uk/government/publications/staying-alert-and-safe-socialdistancing/staying-alert-and-safe-social-distancing (accessed 10 June 2020).

5. Barron E, Bakhai C, Kar P, et al. Type 1 and type 2 diabetes and COVID-19 related mortality in England: a whole population study. Lancet Diabetes En- docrino/ 2020;8(10):813-22. https://doi.org/10.1016/\$2213-8587(20)30272-2

6. NHS England. Coronavirus (COVID-19) in the UK. 2020 (accessed 15 June 2020). https://coronavirus. data.gov.uk/

7. Choudhary P, Wilmot E, Owen K, et al. Letter to the Editor: A roadmap to recovery: $A B C D$ recommedations on risk stratification of adult pateints with diabetes in the post COVID-19 era. Diabet Med 2020;e14462. http://dx.doi.org/10.1111/dme.14462

8. Klonoff DC, Umpierrez GE. Letter to the Editor: COVID-19 in patients with diabetes: risk factors that increase morbidity. Metabolism 2020; 108:154224. https://doi.org/10.1016/j.metabol.2020.154224

9. Academy of Medical Royal Colleges (AOMRC). Principles for reintroducing healthcare services COVID-19. 2020. https://www.aomrc.org.uk/reportsguidance/principles-for-reintroducing-healthcare-services-covid-19/ (accessed 10 June 2020).

10. My Diabetes My Way (MDMY). Type 1 diabetes platform for NHS England launched. 2020 (accessed 13 June 2020). https://www.nhs.uk/apps-library/my-diabetes-my-way/

11. Rodriguez KM. Intrinsic and extrinsic factors affecting patient engagement in diabetes self-management: perspectives of a certified diabetes educator. Clin Ther 2013:35(2):170-8. https://doi.org/10.1016/j.clinthera.2013.01.002

12. Association of British Clinical Diabetologists. COVID-19 (Coronavirus) information for healthcare professionals. 2020. https://abcd.care/coronavirus

13. Association of British Clinical Diabetologists, Society for Endocrinology. Delivering diabetes and endocrine services in acute NHS Trusts during Covid 19 pandemic reset. May 2020. https://abcd.care/sites/abcd.care/files/articles/ABCD-SfE-working-group-V4.1.pdf

14. NHS. Clinical guide for the management of remote consultations and remote working in secondary care during the coronovirus pandemic. 2020. https://www.england.nhs.uk/coronavirus/wp-content/uploads/sites/52/2020/ 03/C0044-Specialty-Guide-Virtual-Working-and-Coronavirus-27-March20.pdf

15. Chaplin S. National Diabetes Audit 2015-2016 shows variations in care processes and targets. Pract Diabetes 2017;34(2):58-60. https://doi.org/ 10.002/pdi.2081

16. NHS England. Care processes and treatment targets, January to December 2019. 2019. https://digital.nhs.uk/data-and-information/publications/statistical/national-diabetes-audit/national-diabetes-audit-quarterly-report-january-to-december-2019 (accessed 15 June 20)

17. Ahn DT. The COVID-19 pandemic: a "Tech"-tonic shift toward virtual diabetes care. J Diabetes Sci Techno/ 2020;14(4):708-9. https://doi.org/ $10.1177 / 1932296820929719$

18. Fatehi F, Martin-Khan M, Smith AC, Russell AW, Gray LC. Patient satisfaction with video teleconsultation in a virtual diabetes outreach clinic. Diabetes Technol Ther 2015;17(1):43-8. https://doi.org/10.1089/dia.2014.0159

19. Greenhalgh T, Vijayaraghavan $S$, Wherton J, et al. Virtual online consultations: advantages and limitations (VOCAL) study. BMJ Open 2016; 6(1):e009388. https://doi.org/10.1136/bmjopen-2015-009388

20. Greenwood DA, Gee PM, Fatkin KJ, Peeples M. A systematic review of reviews evaluating technology-enabled diabetes self-management education and support. J Diabetes Sci Technol 2017;11(5):1015-27. https://doi.org/10.1177/1932296817713506

21. Sharma S, Kerry C, Atkins H, Rayman G. The Ipswich Touch Test: a simple and novel method to screen patients with diabetes at home for increased risk of foot ulceration. Diabet Med 2014;31(9):1100-03. https://doi.org/10.1111/dme.12450

22. Kruger J, Brennan A, Thokala P, et al. The cost-effectiveness of the Dose Adjustment for Normal Eating (DAFNE) structured education programme: an update using the Sheffield Type 1 Diabetes Policy Model. Diabet Med 2013;30(10):1236-44. https://doi.org/10.1111/dme.12270

23. Hopkins D, Lawrence I, Mansell P, et al. Improved biomedical and psychological outcomes 1 year after structured education in flexible insulin therapy for people with type 1 diabetes: the UK DAFNE experience. Diabetes Care 2012;35(8):1638-42. https://doi.org/10.2337/dc11-1579

24. Rayman G, Kar P. Diabetes: GIRFT Programme National Specialty Report. https://www.gettingitrightfirsttime.co.uk/wp-content/uploads/2020/11/ GIRFT-diabetes-report.pdf 